\title{
INVERSE SCATTERING ON THE LINE FOR A GENERALIZED NONLINEAR SCHRÖDINGER EQUATION
}

\author{
Tuncay Aktosun \\ Department of Mathematics and Statistics \\ Mississippi State University \\ Mississippi State, MS 39762, USA \\ aktosun@math.msstate.edu \\ Vassilis G. Papanicolaou and Vassilis Zisis \\ Department of Mathematics \\ National Technical University of Athens \\ Zografou Campus \\ 157 80, Athens, Greece \\ papanico@math.ntua.gr
}

\begin{abstract}
A one-dimensional generalized nonlinear Schrödinger equation is considered, and the corresponding inverse scattering problem is analyzed when the potential is compactly supported and depends on the wave function. The unique recovery of the potential is established from an appropriate set of scattering data.
\end{abstract}

PACS (2003): 2.30.Zz, 3.65.Nk, 43.25.+y

Mathematics Subject Classification (2000): 34A55, 34L25, 34L30

Keywords: nonlinear potential, generalized nonlinear Schrödinger equation, inverse scattering, nonlinear scatterer 


\section{INTRODUCTION}

Consider the nonlinear equation

$$
-u^{\prime \prime}+Q(x, u) u=k^{2} u, \quad x \in \mathbf{R},
$$

where $k$ is a real parameter, the prime denotes the derivative with respect to the spatial variable $x$, and $Q(x, u)$ has the form

$$
Q(x, u)=\sum_{n=0}^{\infty} q_{n}(x) u^{n},
$$

with each $q_{n}(x)$ being real valued, bounded, measurable, supported in $[0, b]$ for a fixed $b>0$, and the series

$$
\sum_{n=0}^{\infty}\left(\sup _{x \in[0, b]}\left|q_{n}(x)\right|\right) u^{n}
$$

being entire in $u$. The assumption that the series in (1.3) is entire is equivalent to assuming that its radius of convergence is infinite, i.e.

$$
\varlimsup_{n \rightarrow+\infty}\left(\sup _{x \in[0, b]}\left|q_{n}(x)\right|\right)^{1 / n}=0 .
$$

Note that such an assumption is stronger than just assuming that $Q(x, u)$ given in $(1.2)$ is entire in $u$.

In this paper we consider the solution to (1.1) satisfying

$$
u(0 ; k)=\varepsilon, \quad u^{\prime}(0 ; k)=-i k \varepsilon .
$$

As shown in Proposition 2.1, a unique solution to (1.1) exists for all $x \in \mathbf{R}$ and $k \in \mathbf{R}$ when $|\varepsilon|$ is sufficiently small. The results given in our paper hold both for real and complex values of $\varepsilon$. We suppress the dependence of $u$ on $\varepsilon$ for simplicity. Since for $n \geq 0$ we assume $q_{n}(x)=0$ when $x \notin[0, b]$, it follows that for each $k \in \mathbf{R} \backslash\{0\}$ the general solution to (1.1) for $x \notin(0, b)$ is a linear combination of $e^{i k x}$ and $e^{-i k x}$. Thus, we have

$$
u(x ; k)=\varepsilon e^{-i k x}, \quad x \leq 0,
$$


which is equivalent to (1.5), and

$$
u(x ; k)=A(k ; \varepsilon) e^{i k x}+B(k ; \varepsilon) e^{-i k x}, \quad x \geq b .
$$

Because of the resemblance with the (linear) Schrödinger equation, we will refer to (1.1) as a generalized nonlinear Schrödinger equation and to $Q(x, u)$ as the (nonlinear) potential. The expressions for $u(x ; k)$ given in (1.6) and (1.7) indicate that we have a scattering problem in hand, where a plane wave is sent from $x=+\infty$ onto the nonhomogeneity $Q(x, u)$, and a part of the wave is transmitted to $x=-\infty$ and a part is reflected back to $x=+\infty$. However, contrary to the linear case, there is in general no energy conservation, i.e. $|B(k ; \varepsilon)|^{2}-|A(k ; \varepsilon)|^{2}$ in general depends on $k$. The linear part $q_{0}(x)$ of the potential $Q(x, u)$ in $(1.2)$ represents the restoring force density in wave propagation governed by (1.1) in the frequency domain. The higher order terms in the potential allow the description of nonlinear reaction of the medium to propagation of elastic waves.

In analogy with the direct and inverse scattering problems for the linear Schrödinger equation, the corresponding problems for (1.1) can be formulated as follows. In the direct problem, given $Q(x, u)$, our task is to determine the "scattering coefficients" $A(k ; \varepsilon)$ and $B(k ; \varepsilon)$ for sufficiently small $|\varepsilon|$. On the other hand, in the inverse scattering problem, given some scattering data related to $A(k ; \varepsilon)$ and $B(k ; \varepsilon)$, the task is to recover $Q(x, u)$. In our paper, we do not study the characterization of the scattering data so that the existence of a corresponding potential is assured; we only discuss the uniqueness and recovery aspects of our inverse problem by assuming that there exists at least one potential corresponding to our scattering coefficients.

The inverse scattering problem analyzed in this paper is analogous to the recent study by Weder [1], where the time-dependent Schrödinger equation with a nonlinear term is investigated and both the linear and nonlinear parts of the potential are recovered from some appropriate scattering data by using a time-domain method. For other related studies of inverse problems on nonlinear equations using time-domain methods, we refer the reader to $[2-5]$ and the references therein.

Our paper is organized as follows. In Section 2 we show that the direct and inverse 
scattering problems for the nonlinear equation (1.1) are equivalent to the corresponding problems for an infinite number of linear equations, and we analyze the basic properties of the scattering data for each linear equation and define the appropriate data set $\mathcal{D}_{n}$ given in (2.16) for each $n \geq 1$. We then solve the inverse scattering problem for each $n \geq 1$ recursively. The solution of the inverse problem when $n=1$ is well known, and in Section 3 we list the basic facts from the case $n=1$ that are needed later on to solve the inverse problems for $n \geq 2$. In Section 4 we show that the solution of the inverse scattering problem for each $n \geq 2$ can be obtained by inverting either of the two integral equations (4.4) and (4.5). In Section 5 we prove the unique invertibility of (4.4) and (4.5) to recover $q_{n-1}(x)$ for each $n \geq 2$, and we summarize the recovery of the nonlinear potential $Q(x, u)$ in terms of the scattering data involving $A(k ; \varepsilon)$ and $B(k ; \varepsilon)$. Finally, in Section 6 , we illustrate the direct and inverse problems for (1.1) with some concrete examples.

\section{PRELIMINARIES}

It is straightforward to verify that $u(x ; k)$ satisfies $(1.1)$ and (1.5) if and only if it satisfies the integral equation

$$
u(x ; k)=\varepsilon e^{-i k x}+\frac{1}{k} \int_{0}^{x} \sin (k(x-t)) Q(t, u(t ; k)) u(t ; k) d t
$$

Proposition 2.1. There exists a constant $\delta>0$ depending only on $Q(x, u)$, but not on $k$ (as long as $k$ is real), such that if $|\varepsilon| \leq \delta$ then a solution $u(x ; k)$ to (1.1) satisfying (1.5) exists for all $x \in \mathbf{R}$, and it is unique.

PROOF: Suppose that a solution $u(x ; k)$ to $(2.1)$ ceases to exist, i.e. blows up, for some $x \in(0, b]$. Then, for any $r$ sufficiently large, there is an $x_{0} \in(0, b]$ such that $\left|u\left(x_{0} ; k\right)\right|=r$ and $|u(x ; k)|<r$ for $x<x_{0}$. Fix one such $r$. Our assumption in (1.4) implies that there is a $C>0$ such that $|Q(x, u)| \leq C$ for $x \in[0, b]$ and $|u| \leq r$. Let us take

$$
|\varepsilon| \leq \delta:=(r / 2) e^{-C b^{2}}
$$


Using $|\sin \theta| \leq|\theta|$ for real $\theta$ and the realness of $k$, for $x<x_{0}$ from (2.1) we get

$$
\begin{aligned}
|u(x ; k)| & \leq|\varepsilon|+\int_{0}^{x}(x-t)|Q(t, u(t ; k))||u(t ; k)| d t \\
& \leq|\varepsilon|+b \int_{0}^{x}|Q(t, u(t ; k))||u(t ; k)| d t .
\end{aligned}
$$

Applying Gronwall's inequality (see, e.g. Prob. 1 in Ch. 1 of [6]) on the last term above, we obtain

$$
|u(x ; k)| \leq|\varepsilon|+b|\varepsilon| \int_{0}^{x}|Q(t, u(t ; k))| \exp \left(b \int_{t}^{x} Q(z, u(z ; k)) d z\right) d t .
$$

Setting $x=x_{0}$ in $(2.3)$, we have

$$
r \leq|\varepsilon|+b|\varepsilon| \int_{0}^{x_{0}} C \exp \left(C b\left(x_{0}-t\right)\right) d t \leq|\varepsilon| e^{C b^{2}},
$$

and a comparison with (2.2) indicates that

$$
0<r \leq|\varepsilon| e^{C b^{2}} \leq(r / 2) e^{-C b^{2}} e^{C b^{2}}=r / 2
$$

which is impossible. Therefore, $u(x ; k)$ does not blow up in $[0, b]$ and hence it exists for all $x \in \mathbf{R}$. The uniqueness of $u(x ; k)$ follows from the Lipschitz property of $Q(x, u)$ with respect to $u$, which, in turn, follows from the analyticity of $Q(x, \cdot)$.

From the conditions in (1.5), with the help of Theorem 8.4 in Sec. 1.8 of [6], when $k$ is real and bounded, $x \in \mathbf{R}$, and $|\varepsilon|$ is sufficiently small, we see that $u$ is analytic in $\varepsilon$ and hence

$$
u(x ; k)=\sum_{n=1}^{\infty} \varepsilon^{n} u_{n}(x ; k)=\varepsilon u_{1}(x ; k)+\varepsilon^{2} u_{2}(x ; k)+\ldots
$$

Note that the $\varepsilon^{0}$-term is absent in (2.4) because (1.5) implies that $u(x ; k) \equiv 0$ if $\varepsilon=0$.

We observe from (1.7) and (2.4) that the analyticity of $u$ in $\varepsilon$ for $x \geq b$ implies that $A(k ; \varepsilon)$ and $B(k ; \varepsilon)$ are analytic in $\varepsilon$ at $\varepsilon=0$ for real, nonzero, and bounded $k$. Thus, we have the expansions

$$
A(k ; \varepsilon)=\sum_{n=1}^{\infty} \varepsilon^{n} A_{n}(k), \quad B(k ; \varepsilon)=\sum_{n=1}^{\infty} \varepsilon^{n} B_{n}(k),
$$


where we emphasize that the $\varepsilon^{0}$-terms are absent. With the help of $(2.1)$ and the expansions (2.4) and (2.5), one can show that the generalized nonlinear Schrödinger equation (1.1), the condition (1.6), and the expression (1.7) are equivalent to an infinite number of scattering problems for linear differential equations. The use of (2.1) allows us to avoid the interchange of the $x$-differentiation and the infinite summation in (2.4). The resulting scattering problems for linear equations are, for $n=1$,

$$
\begin{gathered}
-u_{1}^{\prime \prime}+q_{0}(x) u_{1}=k^{2} u_{1}, \quad x \in \mathbf{R}, \\
u_{1}(x ; k)=e^{-i k x}, \quad x \leq 0, \\
u_{1}(x ; k)=A_{1}(k) e^{i k x}+B_{1}(k) e^{-i k x}, \quad x \geq b,
\end{gathered}
$$

and, for $n \geq 2$,

$$
\begin{gathered}
-u_{n}^{\prime \prime}+q_{0}(x) u_{n}=k^{2} u_{n}-g_{n}(x ; k), \quad x \in \mathbf{R}, \\
u_{n}(x ; k)=0, \quad x \leq 0, \\
u_{n}(x ; k)=A_{n}(k) e^{i k x}+B_{n}(k) e^{-i k x}, \quad x \geq b,
\end{gathered}
$$

where we have defined

$$
\begin{gathered}
g_{n}(x ; k):=q_{n-1}(x) u_{1}(x ; k)^{n}+h_{n}(x ; k), \quad n \geq 2, \\
h_{2}(x ; k):=0 ; \quad h_{n}(x ; k):=\sum_{j=2}^{n-1} C_{j n}(x ; k) q_{j-1}(x), \quad n \geq 3,
\end{gathered}
$$

with $C_{j n}(x ; k)$ for $2 \leq j \leq n-1$ being the coefficient of $\varepsilon^{n}$ in the expansion of

$$
\left[\varepsilon u_{1}(x ; k)+\varepsilon^{2} u_{2}(x ; k)+\cdots+\varepsilon^{n-1} u_{n-1}(x ; k)\right]^{j}
$$

We list the first few $h_{n}(x ; k)$ below:

$$
\begin{gathered}
h_{2}(x ; k)=0, \quad h_{3}(x ; k)=2 u_{1} u_{2} q_{1}, \\
h_{4}(x ; k)=\left(2 u_{1} u_{3}+u_{2}^{2}\right) q_{1}+3 u_{1}^{2} u_{2} q_{2}, \\
h_{5}(x ; k)=2\left(u_{1} u_{4}+u_{2} u_{3}\right) q_{1}+3\left(u_{1} u_{2}^{2}+u_{1}^{2} u_{3}\right) q_{2}+4 u_{1}^{3} u_{2} q_{3} .
\end{gathered}
$$


So far we have established the validity of $u_{n}(x ; k)$ only for real and bounded $k$ [cf. (2.4)] and those of $A_{n}(k)$ and $B_{n}(k)$ [cf. (2.5)] only for real, nonzero and bounded $k$. In the next result, we analyze their analytic extensions in $k$ to the entire complex plane $\mathbf{C}$.

Proposition 2.2. For $n \geq 1$, let $u_{n}(x ; k), A_{n}(k)$, and $B_{n}(k)$ be the quantities given in (2.4) and (2.5). Then, $u_{n}(x ; k)$ for each $x \in \mathbf{R}, k A_{n}(k)$, and $k B_{n}(k)$ are entire in $k$.

PROOF: Since $u_{1}(x ; k)$ satisfies the linear equation (2.6) with the condition in (2.7) and $u_{n}(x ; k)$ for $n \geq 2$ satisfies the linear equation (2.9) with the condition in (2.10), it follows from Theorem 8.4 in Sec. 1.8 of [6] that $u_{n}(x ; k)$ and $u_{n}^{\prime}(x ; k)$ are entire in $k$. From $(2.8)$ and (2.11), for $x \geq b$ and $n \geq 1$ we get

$$
u_{n}(x ; k)=A_{n}(k) e^{i k x}+B_{n}(k) e^{-i k x}, \quad u_{n}^{\prime}(x ; k)=i k A_{n}(k) e^{i k x}-i k B_{n}(k) e^{-i k x},
$$

and hence

$$
k A_{n}(k)=\frac{k u_{n}(x ; k)-i u_{n}^{\prime}(x ; k)}{2} e^{-i k x}, \quad k B_{n}(k)=\frac{k u_{n}(x ; k)+i u_{n}^{\prime}(x ; k)}{2} e^{i k x},
$$

from which we see that $k A_{n}(k)$ and $k B_{n}(k)$ are entire in $k$.

Proposition 2.3. For each fixed $k \in \mathbf{C}$ and all $n \geq 1$, the quantities $u_{n}(\cdot ; k)$ are bounded for $x \in[0, b]$. For each fixed $k \in \mathbf{C}$ and all $n \geq 2$, the quantities $h_{n}(\cdot ; k)$ defined in (2.13) are bounded in $x$ and vanish for $x \notin[0, b]$.

PROOF: The boundedness of $u_{n}(\cdot ; k)$ for $x \in[0, b]$ can be established recursively for $n \geq 1$ by using the fact that each $u_{n}(\cdot ; k)$ is a solution to a linear, ordinary differential equation with appropriate initial conditions at $x=0$ [cf. (2.7) and (2.10)]. It is assumed that $q_{n}(x)$ for each $n \geq 0$ is bounded in $x$ and vanishes outside $[0, b]$. Since $h_{n}(x ; k)$ is a linear combination of $q_{1}, \ldots, q_{n-2}$ with coefficients that are polynomials in $u_{1}, \ldots, u_{n-1}$, it follows that $h_{n}(x ; k)$ is bounded in $x$ and vanishes when $x \notin[0, b]$.

Recall that our aim in this paper is to solve the inverse scattering problem for (1.1), namely to recover $Q(x, u)$ from some scattering data involving $A(k ; \varepsilon)$ and $B(k ; \varepsilon)$. In the light of (1.2) and (2.4)-(2.13), we see that our inverse problem is equivalent to the recovery of the $q_{n-1}(x)$ for each $n \geq 1$ from some data involving $A_{n}(k)$ and $B_{n}(k)$. We will establish 
the uniqueness of the recovery recursively; namely, first $q_{0}(x)$ will be shown to be uniquely recoverable from $\left\{A_{1}(k), B_{1}(k)\right\}$, and then we will prove the unique recovery of $q_{n-1}$ for each $n \geq 2$ from the data set $\mathcal{D}_{n}$, where we have defined

$$
\mathcal{D}_{n}:=\left\{A_{n}(k), B_{n}(k), q_{0}(x), \ldots, q_{n-2}(x)\right\}, \quad n \geq 2 .
$$

As we will see, our uniqueness proof of recovery of $q_{n-1}(x)$ will rely on the values of $A_{n}(k)$ and $B_{n}(k)$ with large complex values of $k$. Recall that we have shown the validity of the solution $u(x ; k)$ to $(1.1)$ with the condition in (1.6) only for real $k$, and those of the scattering coefficients $A(k ; \varepsilon)$ and $B(k ; \varepsilon)$ only for real, nonzero, and bounded $k$. Hence, it is pleasantly surprising that we can prove the uniqueness of recovery of $Q(x, u)$ from $A(k ; \varepsilon)$ and $B(k ; \varepsilon)$ without needing any extensions of the latter quantities to complex $k$ values.

\section{INVERSE SCATTERING TO RECOVER $q_{0}(x)$}

In order to solve the inverse scattering problem for (2.9), we need some basic facts related to (2.6). In this section we list those basic facts and refer the reader to [7-11] for details. Associated with (2.6), let $L$ denote the unique selfadjoint realization of $-d^{2} / d x^{2}+$ $q_{0}(x)$ in $L_{2}(\mathbf{R})$. Even under weaker assumptions on $q_{0}(x)$, namely, when $q_{0}(x)$ is real valued, integrable, and vanishing outside $[0, b]$, the following are known:

(i) $L$ has no positive or zero eigenvalues, it has no singular-continuous spectrum, and its absolutely continuous spectrum consists of $[0,+\infty)$. It has at most a finite number

of (simple) negative eigenvalues, and we will denote the eigenvalues by $-\kappa_{j}^{2}$ for $j=$ $1, \ldots, N$.

(ii) The solution $u_{1}(x ; k)$ to $(2.6)$ satisfying $(2.7)$ is usually known as the Jost solution from the right and sometimes denoted by $f_{\mathrm{r}}(k, x)$. As also indicated in Proposition 2.2, $u_{1}(x ; k)$ is entire in $k$ for each $x \in \mathbf{R}$. From Lemma 1(ii) on page 130 of [8], it follows that for $k \in \overline{\mathbf{C}^{+}}$and $x \in[0, b]$ we have

$$
\left|e^{i k x} u_{1}(x ; k)\right| \leq \exp \left(b \int_{0}^{b}\left|q_{0}(t)\right| d t\right)
$$


and for $k \in \overline{\mathbf{C}^{+}} \backslash\{0\}$ and $x \in[0, b]$ we have

$$
\left|e^{i k x} u_{1}(x ; k)-1\right| \leq \frac{1}{|k|}\left(\int_{0}^{b}\left|q_{0}(t)\right| d t\right) \exp \left(b \int_{0}^{b}\left|q_{0}(z)\right| d z\right)
$$

where we use $\mathbf{C}^{+}$for the upper half complex plane and put $\overline{\mathbf{C}^{+}}:=\mathbf{C}^{+} \cup \mathbf{R}$. Using (3.1) and (3.2), for $k \in \overline{\mathbf{C}^{+}} \backslash\{0\}$ and $x \in[0, b]$ we obtain

$$
\left|e^{i k(n+1) x} u_{1}(x ; k)-1\right| \leq \frac{(n+1)}{|k|}\left(\int_{0}^{b}\left|q_{0}(t)\right| d t\right) \exp \left(b(n+1) \int_{0}^{b}\left|q_{0}(z)\right| d z\right) .
$$

(iii) Another solution to (2.6) which we denote by $v_{1}(x ; k)$, usually known as the Jost solution from the left and sometimes denoted by $f_{1}(k, x)$, satisfies the asymptotic conditions

$$
v_{1}(x ; k)=e^{i k x}[1+o(1)], \quad v_{1}^{\prime}(x ; k)=i k e^{i k x}[1+o(1)], \quad x \rightarrow+\infty .
$$

Because of the compact support property of $q_{0}(x)$ we have

$$
v_{1}(x ; k)=\left\{\begin{array}{l}
B_{1}(k) e^{i k x}-A_{1}(-k) e^{-i k x}, \quad x \leq 0, \\
e^{i k x}, \quad x \geq b .
\end{array}\right.
$$

For each $x \in \mathbf{R}, u_{1}(x ; k)$ is entire in $k$. The inequalities given in (3.1)-(3.3) also hold if we replace $e^{i k x} u_{1}(x ; k)$ in them by $e^{-i k x} v_{1}(x ; k)$, which is a consequence of Lemma 1(i) on page 130 of [8].

(iv) The scattering coefficients $A_{1}(k)$ and $B_{1}(k)$ given in (2.8) are related to the transmission coefficient $T(k)$ and the right reflection coefficient $R(k)$ as $A_{1}(k)=R(k) / T(k)$ and $B_{1}(k)=1 / T(k)$. It is known that $k A_{1}(k)$ and $k B_{1}(k)$ are entire (cf. Proposition 2.2). The quantity $B_{1}(k)$ is nonzero in $\overline{\mathbf{C}^{+}} \backslash\left\{i \kappa_{j}\right\}_{j=1}^{N}$ and has simple zeros at $k=i \kappa_{j}$ for $j=1, \ldots, N$. In the so-called generic case $B_{1}(k)$ has a simple pole at $k=0$, and in the so-called exceptional case $B_{1}(k)$ is continuous at $k=0$.

(v) The potential $q_{0}(x)$ can be recovered from $A_{1}(k) / B_{1}(k)$ known for $k \in \mathbf{R}$ by any of the several methods [7-11]. No bound state data needs to be supplied [12-17] due to the compact support property of $q_{0}(x)$, and in fact the bound state data (i.e. the 
$N$ constants $\kappa_{j}$ and the related norming constants) can be recovered via the unique meromorphic continuation of $A_{1}(k) / B_{1}(k)$ from $k \in \mathbf{R}$ to $k \in \mathbf{C}^{+}$and using its poles and residues. Actually, knowledge of $A_{1}(k) / B_{1}(k)$ in some interval on the real $k$-axis is sufficient to recover $q_{0}(x)$ because of the uniqueness of the meromorphic continuation.

(vi) It is known that $B_{1}(k)$ alone cannot uniquely determine $q_{0}(x)$, and unless $B_{1}(k) \equiv 1$ there are infinitely many potentials corresponding to it. In the absence of bound states, i.e. if $N=0$, the coefficient $A_{1}(k)$ uniquely determines $q_{0}(x)$ provided $1 / B_{1}(k)$ vanishes at $k=0$. Otherwise, there are exactly two distinct potentials corresponding to $A_{1}(k)$. When $N \geq 1$, there is no uniqueness and there are a discrete number of potentials corresponding to $A_{1}(k)$. For details we refer the reader to [18].

(vii) As long as $k^{2}$ is not in the $L_{2}(\mathbf{R})$-spectrum of $L$, i.e. when $k \in \mathbf{C}^{+} \backslash\left\{i \kappa_{j}\right\}_{j=1}^{N}$, the operator $\left(L-k^{2}\right)^{-1}$ is a bounded one from $L_{2}(\mathbf{R})$ to $L_{2}(\mathbf{R})$. The Green's function associated with (2.6), denoted by $G(x, t ; k)$, is defined as the integral kernel of the resolvent $\left(L-k^{2}\right)^{-1}$ in the sense that for any $g \in L_{2}(\mathbf{R})$ we have

$$
\left[\left(L-k^{2}\right)^{-1} g\right](x)=\int_{-\infty}^{\infty} G(x, t ; k) g(t) d t
$$

It follows that, for any $k \in \mathbf{C}^{+} \backslash\left\{i \kappa_{j}\right\}_{j=1}^{N}$ [cf. (i) and (iv) above], we get

$$
-G_{x x}(x, t ; k)+q_{0}(x) G(x, t ; k)=k^{2} G(x, t ; k)+\delta(x-t),
$$

where $\delta(\cdot)$ is the Dirac delta distribution. With the help of the Wronskian identity

$$
2 i k B_{1}(k)=v_{1}^{\prime}(x ; k) u_{1}(x ; k)-v_{1}(x ; k) u_{1}^{\prime}(x ; k),
$$

it can be verified that

$$
G(x, t ; k)= \begin{cases}-\frac{1}{2 i k B_{1}(k)} v_{1}(t ; k) u_{1}(x ; k), & x<t, \\ -\frac{1}{2 i k B_{1}(k)} v_{1}(x ; k) u_{1}(t ; k), & x>t .\end{cases}
$$

It is seen from (2.7), the second line in (3.4), and (3.7) that, when $k \in \mathbf{C}^{+} \backslash\left\{i \kappa_{j}\right\}_{j=1}^{N}$ the Green's function $G(x, t ; k)$ decays exponentially as $x \rightarrow \pm \infty$, and it is the only solution to (3.6) having this property. 


\section{INVERSE SCATTERING FOR $q_{n-1}(x)$ WITH $n \geq 2$}

In this section we show that the recovery of $q_{n-1}(x)$ for each $n \geq 2$ is equivalent to inverting the integral equation given in (4.4) below or the one given in (4.5). Having seen in the previous section that $q_{0}(x)$ can be recovered uniquely from $\left\{A_{1}(k), B_{1}(k)\right\}$, we proceed recursively and prove the unique recovery of $q_{n-1}(x)$ from the data set $\mathcal{D}_{n}$ defined in $(2.16)$.

With the help of (2.6)-(2.11), we define

$$
y_{n}(x ; k):=u_{n}(x ; k)-\frac{B_{n}(k)}{B_{1}(k)} u_{1}(x ; k), \quad n \geq 2 .
$$

The relevant properties of $y_{n}(x ; k)$ are analyzed next.

Proposition 4.1. Let $y_{n}(x ; k)$ be the quantity defined in (4.1). Then:

(i) For each $k \in \mathbf{C}^{+} \backslash\left\{i \kappa_{j}\right\}_{j=1}^{N}$, where $\left\{i \kappa_{j}\right\}_{j=1}^{N}$ is the set of zeros of $B_{1}(k)$ in $\mathbf{C}^{+}$, the quantity $y_{n}(x ; k)$ is a solution to (2.9), and it is the only solution belonging to $L_{2}(\mathbf{R})$ in $x$. In fact, $y_{n}(\cdot ; k)$ decays exponentially as $x \rightarrow \pm \infty$.

(ii) $y_{n}(x ; k)$ satisfies

$$
y_{n}(x ; k)=\left\{\begin{array}{l}
-\frac{B_{n}(k)}{B_{1}(k)} e^{-i k x}, \quad x \leq 0, \\
{\left[A_{n}(k)-\frac{B_{n}(k)}{B_{1}(k)} A_{1}(k)\right] e^{i k x}, \quad x \geq b .}
\end{array}\right.
$$

(iii) In terms of the nonhomogeneous term $g_{n}(x ; k)$ defined in (2.12) and the Green's function $G(x, t ; k)$ given in (3.7), we have

$$
y_{n}(x ; k)=-\int_{-\infty}^{\infty} G(x, t ; k) g_{n}(t ; k) d t, \quad k \in \mathbf{C}^{+} \backslash\left\{i \kappa_{j}\right\}_{j=1}^{N} .
$$

PROOF: First, from Propositions 2.2 and 2.3 and the fact that the only zeros of $B_{1}(k)$ in $\mathbf{C}^{+}$occur at $k=i \kappa_{j}$ for $j=1, \ldots, N$, it follows that $y_{n}(x ; k)$ is well defined for each $k \in \mathbf{C}^{+} \backslash\left\{i \kappa_{j}\right\}_{j=1}^{N}$. Note that $y_{n}(x ; k)$ solves $(2.9)$ because $u_{n}(x ; k)$ is a solution to the same equation and $u_{1}(x ; k)$ is a solution to the corresponding homogeneous equation. We 
obtain (4.2) directly from (2.7), (2.8), (2.10), and (2.11). For each fixed $k \in \mathbf{C}^{+} \backslash\left\{i \kappa_{j}\right\}_{j=1}^{N}$, since $e^{-i k x}$ and $e^{i k x}$ decay exponentially as $x \rightarrow-\infty$ and as $x \rightarrow+\infty$, respectively, we get the $L_{2}(\mathbf{R})$-property of $y_{n}(\cdot ; k)$ stated in $(\mathrm{i})$. Note that $y_{n}(x ; k)$ is the only $L_{2}(\mathbf{R})$ solution to (2.9) because the difference with any other $L_{2}(\mathbf{R})$-solution must satisfy (2.6); however, as indicated in (i) of Section 3, the only $L_{2}(\mathbf{R})$-solutions to (2.6) occur when $k=i \kappa_{j}$ for $j=1, \ldots, N$. Thus, we have proved (i) and (ii). From the properties listed in Proposition 2.3, it follows that $g_{n}(\cdot ; k)$ is bounded and supported in $[0, b]$ and hence belongs to $L_{2}(\mathbf{R})$. In terms of the operator $L$ defined in Section $3, y_{n}(x ; k)$ satisfies $\left(L-k^{2}\right) y_{n}=-g_{n}$, and hence with the help of (3.5) we obtain (4.3).

In the next theorem we present the main integral equations from which $q_{n-1}(x)$ with $n \geq 2$ will be recovered.

Theorem 4.2. For each $n \geq 2$, the potential $q_{n-1}(x)$ satisfies

$$
\begin{gathered}
\int_{0}^{b} v_{1}(t ; k) u_{1}(t ; k)^{n} q_{n-1}(t) d t=E_{n}(k), \quad k \in \mathbf{C}, \\
\int_{0}^{b} u_{1}(t ; k)^{n+1} q_{n-1}(t) d t=F_{n}(k), \quad k \in \mathbf{C},
\end{gathered}
$$

where $E_{n}(k)$ and $F_{n}(k)$ are completely determined by the data $\mathcal{D}_{n}$ defined in (2.16), and they are given as

$$
\begin{gathered}
E_{n}(k):=-2 i k B_{n}(k)-\int_{0}^{b} v_{1}(t ; k) h_{n}(t ; k) d t \\
F_{n}(k):=2 i k\left[B_{1}(k) A_{n}(k)-A_{1}(k) B_{n}(k)\right]-\int_{0}^{b} u_{1}(t ; k) h_{n}(t ; k) d t .
\end{gathered}
$$

PROOF: Using (2.7) and (3.4) in (3.7), we get

$$
G(x, t ; k)=\left\{\begin{array}{lr}
-\frac{1}{2 i k B_{1}(k)} e^{-i k x} v_{1}(t ; k), & x \leq \min \{0, t\}, \\
-\frac{1}{2 i k B_{1}(k)} e^{i k x} u_{1}(t ; k), & x \geq \max \{b, t\} .
\end{array}\right.
$$

Using (4.8) on the right hand side of (4.3), we obtain

$$
y_{n}(x ; k)= \begin{cases}\frac{1}{2 i k B_{1}(k)} e^{-i k x} \int_{0}^{b} v_{1}(t ; k) g_{n}(t ; k) d t, & x \leq 0, \\ \frac{1}{2 i k B_{1}(k)} e^{i k x} \int_{0}^{b} u_{1}(t ; k) g_{n}(t ; k) d t, & x \geq b .\end{cases}
$$


Comparing (4.9) with (4.2), we see that

$$
\begin{gathered}
2 i k B_{n}(k)=-\int_{0}^{b} v_{1}(t ; k) g_{n}(t ; k) d t, \quad n \geq 2, \\
2 i k\left[B_{1}(k) A_{n}(k)-A_{1}(k) B_{n}(k)\right]=\int_{0}^{b} u_{1}(t ; k) g_{n}(t ; k) d t, \quad n \geq 2 .
\end{gathered}
$$

With the help of (2.12), we rewrite (4.10) and (4.11) as (4.4) and (4.5), respectively. Even though we have derived (4.4) and (4.5) only for $k \in \mathbf{C}^{+} \backslash\left\{i \kappa_{j}\right\}_{j=1}^{N}$, with the help analytic extensions indicated in Proposition 2.2, we see that the former is actually valid for $k \in \mathbf{C}$ and the latter for $k \in \mathbf{C} \backslash\{0\}$. Furthermore, the analytic extension to $k=0$ for (4.5) is proved by showing that the quantity $k\left[B_{1}(k) A_{n}(k)-A_{1}(k) B_{n}(k)\right]$ appearing in (4.7) is continuous at $k=0$. In order to see this, with the help of (2.15), as $k \rightarrow 0$ in $\mathbf{C}$ we obtain

$$
2 i k\left[B_{1}(k) A_{n}(k)-A_{1}(k) B_{n}(k)\right]=u_{n}^{\prime}(x ; k)\left[A_{1}(k)+B_{1}(k)\right]+O(1) .
$$

Although each of $A_{1}(k)$ and $B_{1}(k)$ may have a simple pole at $k=0$, their sum $A_{1}(k)+B_{1}(k)$ is continuous at $k=0$ because (2.8) implies

$$
A_{1}(k)+B_{1}(k)=e^{-i k x} u_{1}(x ; k)+k B_{1}(k) \frac{1-e^{-2 i k x}}{k}, \quad x \geq b,
$$

and the right hand side is $O(1)$ as $k \rightarrow 0$ in $\mathbf{C}$.

With the help of Theorem 4.2, we see that our inverse scattering problem of recovery of $q_{n-1}(x)$ for each $n \geq 2$ can be stated as follows: Given $E_{n}(k)$ or $F_{n}(k)$, determine $q_{n-1}(x)$ for $x \in[0, b]$ by inverting either (4.4) or (4.5).

From (2.13) and (4.6) it is seen that the data set $\left\{B_{n}(k), q_{0}(x), \ldots, q_{n-1}(x)\right\}$ uniquely determines all the quantities given in (4.4) except for $q_{n-1}(x)$ there, and hence one can use (4.4) to determine $q_{n-1}(x)$ without needing $A_{n}(k)$ in the data.

\section{UNIQUE RECOVERY OF $q_{n-1}(x)$ WITH $n \geq 2$}

In this section we show that $q_{n-1}(x)$ with $n \geq 2$ can be recovered by inverting either (4.4) or (4.5). We will present the inversion only for (4.5) because the inversion of (4.4) is similar. From (3.3) we see that we can write

$$
u_{1}(t ; k)^{n+1}=e^{-i k(n+1) t}\left[1+\frac{U(t ; k)}{k}\right], \quad x \in[0, b], \quad k \in \overline{\mathbf{C}^{+}} \backslash\{0\},
$$


where $U(t ; k)$ is uniformly bounded for $x \in[0, b]$ and $k \in \overline{\mathbf{C}^{+}}$; i.e.

$$
|U(x ; k)| \leq M, \quad x \in[0, b], \quad k \in \overline{\mathbf{C}^{+}}
$$

where $M$ is independent of $x$ and $k$. Let us separate the real and imaginary parts of the complex $k$ variable in $\mathbf{C}^{+}$, discretize the former, and keep the latter as a fixed positive parameter by writing

$$
k_{m}=\frac{2 \pi m}{(n+1) b}+i \xi, \quad m \in \mathbf{Z} \text {. }
$$

Let us evaluate (4.5) at $k=k_{m}$ and write the resulting equation in the operator form as $K \phi=p$, or equivalently as

$$
\int_{0}^{b} K(m, t) \phi(t) d t=p(m), \quad m \in \mathbf{Z},
$$

where we have defined [cf. (5.1) and (5.3)]

$$
\begin{gathered}
K(m, t):=e^{-\xi(n+1) t} u_{1}(t ; k)^{n+1}=e^{-2 \pi i m t / b}\left[1+\frac{U\left(t ; k_{m}\right)}{2 \pi m(n+1)^{-1} b^{-1}+i \xi}\right] \\
\phi(t):=e^{\xi(n+1) t} q_{n-1}(t), \\
p(m):=F_{n}\left(k_{m}\right) .
\end{gathered}
$$

Note that we suppress the dependence on $n$ and $\xi$ because the inversion of (4.5) will be done at fixed values of $n$ and $\xi$.

When $q_{0}(x) \equiv 0$, we have $u_{1}(t ; k) \equiv e^{-i k t}$ and $U(t ; k) \equiv 0$; in that case the operator $K$ reduces to $K_{0}$, which is given by [cf. (5.5)]

$$
\left(K_{0} g\right)(m)=\int_{0}^{b} K_{0}(m, t) \phi(t) d t=\int_{0}^{b} e^{-2 \pi i m t / b} \phi(t) d t .
$$

The properties of $K$ and $K_{0}$ are analyzed next.

Theorem 5.1. The operators $K$ and $K_{0}$ defined in (5.4) and (5.8), respectively, satisfy the following:

(i) $K_{0}$ maps $L_{2}(0, b)$ into $l_{2}(\mathbf{Z})$, and its operator norm is given by $\left\|K_{0}\right\|=\sqrt{b}$. 
(ii) $K_{0}^{-1}$ exists as a map from $l_{2}(\mathbf{Z})$ into $L_{2}(0, b)$, and its operator norm is given by $\left\|K_{0}^{-1}\right\|=1 / \sqrt{b}$.

(iii) $K-K_{0}$ maps $L_{2}(0, b)$ into $l_{2}(\mathbf{Z})$. The operator norm of $K-K_{0}$ satisfies $\left\|K-K_{0}\right\| \leq$ $\sqrt{s(\xi)}$, where $s(\xi)$ is a monotone decreasing function on $\xi \in(0,+\infty)$ vanishing at infinity.

(iv) There exists $\xi_{0}>0$, determined by $n, b$, and $\int_{0}^{b}\left|q_{0}(t)\right| d t$ alone, such that the inverse operator $K^{-1}$ exists for any $\xi>\xi_{0}$.

PROOF: For the proof of (i), note that $K_{0}$ is the standard Fourier series operator and hence it maps $L_{2}(0, b)$ into $l_{2}(\mathbf{Z})$. With the help of the completeness relation

$$
\sum_{m \in \mathbf{Z}} e^{2 \pi i m t / b} e^{-2 \pi i m x / b}=b \cdot \delta(t-x), \quad t, x \in \mathbf{R},
$$

using the definition of the operator norm, from (5.8) we obtain $\left\|K_{0}\right\|=\sqrt{b}$. As for (ii), the inverse Fourier series operator $K_{0}^{-1}$ is given by

$$
\left(K_{0}^{-1} h\right)(t)=\frac{1}{b} \sum_{m \in \mathbf{Z}} h(m) e^{2 \pi i m t / b},
$$

and its operator norm is computed in a straightforward manner with the help of

$$
\int_{0}^{b} e^{2 \pi i m t / b} e^{-2 \pi i j t / b} d t=b \cdot \delta_{m j}, \quad m, j \in \mathbf{Z}
$$

where $\delta_{m j}$ denotes the Kronecker delta, yielding $\left\|K_{0}^{-1}\right\|=1 / \sqrt{b}$. As for (iii), note that the square of the operator norm of $K-K_{0}$ is defined as

$$
\left\|K-K_{0}\right\|^{2}:=\sup _{\|g\|=1}\left\|\left(K-K_{0}\right) g\right\|^{2}=\sup _{\|g\|=1} \sum_{m \in \mathbf{Z}}\left|\left[\left(K-K_{0}\right) g\right](m)\right|^{2},
$$

where $\|g\|$ denotes the norm of $g$ in $L_{2}(0, b)$. With the help of (5.3)-(5.5) and (5.8) we get

$$
\left\|K-K_{0}\right\|^{2}=\sup _{\|g\|^{2}=1} \sum_{m \in \mathbf{Z}}\left|\int_{0}^{b} \frac{U\left(t ; k_{m}\right)}{2 \pi m(n+1)^{-1} b^{-1}+i \xi} \phi(t) d t\right|^{2} .
$$

Using the Cauchy-Schwarz inequality on (5.10), $\|g\|^{2}=1$, and (5.2), we obtain

$$
\begin{aligned}
\left\|K-K_{0}\right\|^{2} & \leq \sum_{m \in \mathbf{Z}} \int_{0}^{b}\left|\frac{U\left(t ; k_{m}\right)}{2 \pi m(n+1)^{-1} b^{-1}+i \xi}\right|^{2} d t \\
& \leq M^{2} b \sum_{m \in \mathbf{Z}} \frac{1}{4 \pi^{2} m^{2}(n+1)^{-2} b^{-2}+\xi^{2}},
\end{aligned}
$$


and since the summation in the last term can be evaluated in a closed form, we obtain

$$
\left\|K-K_{0}\right\|^{2} \leq \frac{M^{2} b^{2}(n+1)}{2 \xi} \operatorname{coth}\left(\frac{(n+1) b \xi}{2}\right), \quad \xi>0, \quad n \geq 2 .
$$

Note that $\operatorname{coth}(x)$ is monotone decreasing on $x \in(0,+\infty)$. Using (3.3), (5.2), and (5.11), we see that $\left\|K-K_{0}\right\| \leq \sqrt{s(\xi)}$, where

$$
s(\xi):=\frac{b^{2}(n+1)^{3}}{2 \xi}\left(\int_{0}^{b}\left|q_{0}(x)\right| d x\right)^{2} \operatorname{coth}\left(\frac{(n+1) b \xi}{2}\right) \exp \left(2 b(n+1) \int_{0}^{b}\left|q_{0}(x)\right| d x\right)
$$

Next, we need to prove (iv). Writing

$$
K=K_{0}+\left(K-K_{0}\right)=K_{0}\left[I+K_{0}^{-1}\left(K-K_{0}\right)\right]
$$

we see that $K^{-1}$ can be evaluated as a Neumann series

$$
K^{-1}=\left[I+K_{0}^{-1}\left(K-K_{0}\right)\right]^{-1} K_{0}^{-1}=\sum_{j=0}^{\infty}(-1)^{j}\left[K_{0}^{-1}\left(K-K_{0}\right)\right]^{j} K_{0}^{-1}
$$

whose convergence is assured by choosing $\left\|K_{0}^{-1}\right\|\left\|\left(K-K_{0}\right)\right\|<1$. From (ii) and (iii), we see that this is achieved by choosing $\xi_{0}$ as the unique solution to $s\left(\xi_{0}\right)=b$. Then, for any $\xi>\xi_{0}$, the existence of $K^{-1}$ is assured.

Having shown that $K$ is invertible for sufficiently large $\xi$ values, we can recover $q_{n-1}(x)$ with the help of (5.4), (5.6), and (5.7) as

$$
q_{n-1}(x)=e^{-\xi(n+1) x}\left(K^{-1} F_{n}\right)(x), \quad x \in[0, b], \quad n \geq 2
$$

Let us now summarize the reconstruction in the solution of the inverse scattering problem for (1.1). Assume that we are given the scattering data consisting of $A(k ; \varepsilon)$ and $B(k ; \varepsilon)$ for all $k$ in some subinterval of the positive $k$-axis and for all $|\varepsilon| \leq \delta$, where $\delta$ is some positive number (no matter how small). Our aim is to recover the potential $Q(x, u)$ corresponding to the given scattering data.

(i) Using (2.5), obtain $A_{n}(k)$ and $B_{n}(k)$ for $n \geq 1$. Note that $k A_{n}(k)$ and $k B_{n}(k)$ have entire extensions to $\mathbf{C}$. 
(ii) Recover $q_{0}(x)$ and $u_{1}(x ; k)$ from $\left\{A_{1}(k), B_{1}(k)\right\}$ by using any one of the available inversion methods described in (v) of Section 3.

(iii) Recover $q_{n-1}(x)$ and $u_{n}(x ; k)$ for $n \geq 2$ by using the data $\mathcal{D}_{n}$ given in $(2.16)$ in a recursive way. The recovery of $q_{n-1}(x)$ is achieved by inverting either (4.4) or (4.5). The recovery of $u_{n}(x ; k)$ amounts to solving the linear equation $(2.9)$ with the condition in (2.10).

(iv) Having recovered all the $q_{n-1}(x)$ for $n \geq 1$, we obtain $Q(x, u)$ via (1.2).

The uniqueness of the recovery of $Q(x, u)$ from the data $\{A(k ; \varepsilon), B(k ; \varepsilon)\}$ is summarized next.

Theorem 5.2. Consider the data consisting of $A(k ; \varepsilon)$ and $B(k ; \varepsilon)$ given for all $k$ in some subinterval of the positive $k$-axis and for all $|\varepsilon| \leq \delta$, where $\delta$ is some positive number (no matter how small). Further assume that there exists a potential $Q(x, u)$ corresponding to this data. Then $Q(x, u)$ is the only potential corresponding to that data.

\section{EXAMPLES}

In this section we illustrate the direct and inverse problems for (1.1) in a special case with some explicit examples. Assume that $Q(x, u)$ given in (1.2) has the form $Q(x, u)=$ $q_{2}(x) u^{2}$, i.e. $q_{j}(x) \equiv 0$ for $j \geq 0$ except when $j=2$. From $(2.6)-(2.8)$ we get $u_{1}(x ; k)=$

$e^{-i k x}$ for $x \in \mathbf{R}, A_{1}(k)=0, B_{1}(k)=1$. From (2.9)-(2.13) with $n=2$, we obtain $u_{2}(x ; k)=$ 0 for $x \in \mathbf{R}, A_{2}(k)=B_{2}(k)=0$. Using (2.9)-(2.11) with $n=3$, we see that $u_{3}(x ; k)$ satisfies

$$
u_{3}^{\prime \prime}(x ; k)+k^{2} u_{3}(x ; k)=q_{2}(x) e^{-3 i k x}, \quad x \in \mathbf{R}
$$

with the initial conditions $u_{3}(0 ; k)=u_{3}^{\prime}(0 ; k)=0$. Using variation of parameters, we get

$$
u_{3}(x ; k)=\frac{1}{2 i k} e^{i k x} \int_{0}^{x} q_{2}(t) e^{-4 i k t} d t-\frac{1}{2 i k} e^{-i k x} \int_{0}^{x} q_{2}(t) e^{-2 i k t} d t, \quad x \in \mathbf{R} .
$$

Comparing (6.1) with (2.11) we see that

$$
A_{3}(k)=\frac{1}{2 i k} \int_{0}^{b} q_{2}(t) e^{-4 i k t} d t, \quad B_{3}(k)=-\frac{1}{2 i k} \int_{0}^{b} q_{2}(t) e^{-2 i k t} d t .
$$


Thus, we see that $A_{3}(k)$ and $B_{3}(k)$ are related to each other as $A_{3}(k)=-2 B_{3}(2 k)$. With the help of $\int_{-\infty}^{\infty} e^{i k \alpha} d k=2 \pi \delta(\alpha)$, the potential $q_{2}(x)$ is uniquely recovered from $A_{3}(k)$ or $B_{3}(k)$ given in $(6.2)$ as

$$
q_{2}(x)=\frac{4 i}{\pi} \int_{-\infty}^{\infty} k A_{3}(k) e^{4 i k x} d k=-\frac{2 i}{\pi} \int_{-\infty}^{\infty} k B_{3}(k) e^{2 i k x} d k
$$

Alternatively, $q_{2}(x)$ can be recovered by using the method of Section 5 as follows. Let us suppose that we are given $A_{3}(k)$. Using (2.14) and (4.7) we see that $F_{3}(k)=2 i k A_{3}(k)$. Thus, with the help of (5.3) and (5.7) we get

$$
p(m)=(i m \pi / b-2 \xi) A_{3}(i \xi+m \pi / 2 b), \quad m \in \mathbf{Z} .
$$

Since $q_{0}(x) \equiv 0$, we have $K=K_{0}$, where $K$ and $K_{0}$ are the operators appearing in (5.4) and (5.8), respectively. Thus, for any $\xi>0$, with the help of (5.9) and (5.12), we construct $q_{2}(x)$ explicitly on $[0, b]$ via

$$
q_{2}(x)=\frac{1}{b^{2}} e^{-\xi(n+1) x} \sum_{m \in \mathbf{Z}} e^{2 \pi i m x / b}(i m \pi-2 b \xi) A_{3}(i \xi+m \pi / 2 b)
$$

In particular, if $q_{2}(x)$ is a constant on the interval $[0, b]$, say $q_{2}(x)=\gamma$, from $(6.2)$ we get

$$
A_{3}(k)=-\frac{\gamma}{8 k^{2}}\left(1-e^{-4 i k b}\right), \quad B_{3}(k)=\frac{\gamma}{4 k^{2}}\left(1-e^{-2 i k b}\right) .
$$

Conversely, if $A_{3}(k)$ and $B_{3}(k)$ are as in (6.5), then with the help of

$$
\int_{-\infty}^{\infty} \frac{e^{i k a}}{k} d k=i \pi \operatorname{sgn}(a)
$$

where $\operatorname{sgn}(a)$ denotes the signature function, we recover $q_{2}(x)=\gamma$ via (6.3). Alternatively, $q_{2}(x)$ can be recovered by using (6.4).

In another particular case, in which $q_{2}(x)=e^{\alpha x}$ on the interval $[0, b]$, where $\alpha$ is a constant, from (6.2) we get

$$
A_{3}(k)=\frac{e^{(\alpha-4 i k) b}-1}{2 i k(\alpha-4 i k)}, \quad B_{3}(k)=-\frac{e^{(\alpha-2 i k) b}-1}{2 i k(\alpha-2 i k)} .
$$


Conversely, if $A_{3}(k)$ and $B_{3}(k)$ are as in (6.6), then we recover $q_{2}(x)=e^{\alpha x}$ via (6.3) with the help of a contour integration. Alternatively, we can recover $q_{2}(x)$ by using (6.4).

Acknowledgment. The research leading to this article was supported in part by the National Science Foundation under grant DMS-0204437 and the Department of Energy under grant DE-FG02-01ER45951.

\section{References}

[1] R. Weder, Inverse scattering for the non-linear Schrödinger equation: Reconstruction of the potential and the non-linearity, Math. Meth. Appl. Sci. 24, 245-254 (2001).

[2] R. Weder, Inverse scattering on the line for the nonlinear Klein-Gordon equation with a potential, J. Math. Anal. Appl. 252, 102-123 (2000).

[3] C. S. Morawetz and W. A. Strauss, On a nonlinear scattering operator, Commun. Pure Appl. Math. 26, 47-54 (1973).

[4] A. Bachelot, Inverse scattering problem for the nonlinear Klein-Gordon equation, In: C. Bardos et al. (eds.), Contributions to nonlinear partial differential equations (Madrid, 1981), Res. Notes in Math. 89, Pitman, Boston, MA, 1983, pp. 7-15.

[5] R. Weder, Inverse scattering for the nonlinear Schrödinger equation. II. Reconstruction of the potential and the nonlinearity in the multidimensional case, Proc. Amer. Math. Soc. 129, 3637-3645 (2001).

[6] E. A. Coddington and N. Levinson, Theory of ordinary differential equations, Robert E. Krieger Publ. Co., Malabar, Florida, 1987.

[7] L. D. Faddeev, Properties of the S-matrix of the one-dimensional Schrödinger equation, Amer. Math. Soc. Transl. (ser. 2) 65, 139-166 (1967).

[8] P. Deift and E. Trubowitz, Inverse scattering on the line, Commun. Pure Appl. Math. 32, 121-251 (1979).

[9] V. A. Marchenko, Sturm-Liouville operators and applications, Birkhäuser, Basel, 1986. 
[10] K. Chadan and P. C. Sabatier, Inverse problems in quantum scattering theory, 2nd ed., Springer, New York, 1989.

[11] T. Aktosun and M. Klaus, Chapter 2.2.4, Inverse theory: problem on the line, In: E. R. Pike and P. C. Sabatier (eds.), Scattering, Academic Press, London, 2001, pp. 770-785.

[12] N. N. Novikova and V. M. Markushevich, Uniqueness of the solution of the onedimensional problem of scattering for potentials located on the positive semiaxis, Comput. Seismology 18, 164-172 (1987).

[13] T. Aktosun, M. Klaus, and C. van der Mee C, On the Riemann-Hilbert problem for the one-dimensional Schrödinger equation, J. Math. Phys. 34, 2651-2690 (1993).

[14] T. Aktosun, Bound states and inverse scattering for the Schrödinger equation in one dimension, J. Math. Phys. 35, 6231-6236 (1994).

[15] B. Grébert and R. Weder, Reconstruction of a potential on the line that is a priori known on the half line, SIAM J. Appl. Math. 55, 242-254 (1995).

[16] T. Aktosun, Inverse Schrödinger scattering on the line with partial knowledge of the potential, SIAM J. Appl. Math. 56, 219-231 (1996).

[17] F. Gesztesy and B. Simon, Inverse spectral analysis with partial information on the potential. I. The case of an a.c. component in the spectrum, Helv. Phys. Acta 70, 66-71 (1997).

[18] T. Aktosun and V. G. Papanicolaou, Recovery of a potential from the ratio of reflection and transmission coefficients, J. Math. Phys. 44, 4875-4883 (2003). 